\title{
Commensurability classes of 2-bridge knot complements
}

\author{
ALAN W REID \\ GeNEVIEVE S WALSH
}

\begin{abstract}
We show that a hyperbolic 2-bridge knot complement is the unique knot complement in its commensurability class. We also discuss constructions of commensurable hyperbolic knot complements and put forth a conjecture on the number of hyperbolic knot complements in a commensurability class.
\end{abstract}

57M25, 57M10; 57M27

\section{Introduction}

Recall that two hyperbolic 3-manifolds $M_{1}=\mathbf{H}^{3} / \Gamma_{1}$ and $M_{2}=\mathbf{H}^{3} / \Gamma_{2}$ are commensurable if they have homeomorphic finite sheeted covering spaces. In terms of the groups, this is equivalent to $\Gamma_{1}$ and some conjugate of $\Gamma_{2}$ in $\operatorname{Isom}\left(\mathbf{H}^{3}\right)$ having a common finite index subgroup. Proving that two hyperbolic 3-manifolds are commensurable (or not commensurable) is in general a difficult problem. The most useful techniques at present are algebraic, for example, the invariant trace-field (see Maclachlan and Reid [13, Chapter 3]). An algorithm to determine when two nonarithmetic cusped hyperbolic 3-manifolds are commensurable is given by Goodman, Heard and Hodgson [8].

In this paper we investigate commensurability of hyperbolic knot complements in $S^{3}$. Our main result is:

Theorem 1.1 Let $K$ be a hyperbolic 2-bridge knot. Then $S^{3} \backslash K$ is not commensurable with the complement of any other knot in $S^{3}$.

Previous work in this direction was done by the first author in [20], where it is shown that the figure-eight knot is the only knot in $S^{3}$ whose complement is arithmetic. In addition, it is known that no two hyperbolic twist knot complements are commensurable by Hoste and Shanahan [10]. On the other hand, there are hyperbolic knot complements in $S^{3}$ with more than one knot complement in the commensurability class. A detailed discussion of this is given in Section 5.

A corollary of our main theorem is the following result which is a direct consequence of Corollary 1.4 of Schwartz [25]. 
Corollary 1.2 Let $K$ be a hyperbolic 2-bridge knot in $S^{3}$ and $K^{\prime}$ any knot in $S^{3}$. If $\pi_{1}\left(S^{3} \backslash K\right)$ and $\pi_{1}\left(S^{3} \backslash K^{\prime}\right)$ are quasi-isometric, then $K$ and $K^{\prime}$ are equivalent.

Acknowledgements The authors wish to thank Michel Boileau, Cameron Gordon and Walter Neumann for some useful conversations on matters related to this work. The first author also wishes to thank the Institute for Advanced Study and the Universidade Federal do Ceará, Fortaleza for their hospitality during the preparation of this work. The first author was partially supported by the NSF.

\section{Preliminaries}

We begin by recalling some terminology and results that will be needed. Henceforth, unless otherwise stated knot complement will always refer to a knot complement in $S^{3}$.

\subsection{Hidden symmetries}

Let $\Gamma$ be a Kleinian group of finite co-volume. The commensurator of $\Gamma$ is the group

$$
C(\Gamma)=\left\{g \in \operatorname{Isom}\left(\mathbf{H}^{3}\right):\left[\Gamma: \Gamma \cap g^{-1} \Gamma g\right]<\infty\right\} .
$$

We denote by $C^{+}(\Gamma)$ the subgroup of $C(\Gamma)$ of index at most 2 that consists of orientation-preserving isometries. It is a fundamental result of Margulis [14] that $C^{+}(\Gamma)$ is a Kleinian group if and only if $\Gamma$ is nonarithmetic, and moreover, in this case, $C^{+}(\Gamma)$ is the unique maximal element in the $\operatorname{PSL}(2, \mathbb{C})$ commensurability class of $\Gamma$.

Note that the normalizer of $\Gamma$ in $\operatorname{PSL}(2, \mathbb{C})$, which we shall denote by $N^{+}(\Gamma)$, is a subgroup of $C^{+}(\Gamma)$. In the case when $\Gamma$ corresponds to the faithful discrete representation of $\pi_{1}\left(S^{3} \backslash K\right)$ it is often the case that $N^{+}(\Gamma)=C^{+}(\Gamma)$. Before we give a more precise discussion of this, we recall some of Neumann and Reid [17]. Henceforth, any knot will be assumed hyperbolic and distinct from the figure-eight knot.

Assume that $S^{3} \backslash K=\mathbf{H}^{3} / \Gamma_{K} . K$ is said to have hidden symmetries if $C^{+}\left(\Gamma_{K}\right)$ properly contains $N(K)=N^{+}\left(\Gamma_{K}\right)$. We will make use of the following result from [17], which requires one more piece of terminology.

Let $S^{2}(2,4,4), S^{2}(2,3,6)$ and $S^{2}(3,3,3)$ denote the 2-dimensional orbifolds which are 2 -spheres with 3 cone points and cone angles $(\pi, \pi / 2, \pi / 2),(\pi, 2 \pi / 3, \pi / 3)$ and $(2 \pi / 3,2 \pi / 3,2 \pi / 3)$ respectively. These are called Euclidean turnovers. In addition, 
we let $S^{2}(2,2,2,2)$ denote the 2 -dimensional orbifold which is a 2 -sphere with 4 cone points all of cone angle $\pi$.

If $X$ is an orientable, noncompact finite volume hyperbolic 3-orbifold, then a cusp of $X$ has the form $Q \times[0, \infty)$, where $Q$ is a Euclidean orbifold. The cusp is said to be rigid if $Q$ is a Euclidean turnover.

Proposition 2.1 [17, Proposition 9.1] The following are equivalent for a hyperbolic knot $K$ other than the figure eight knot complement:

(i) $K$ has hidden symmetries.

(ii) The orientable commensurator quotient $\mathbf{H}^{3} / C^{+}\left(\Gamma_{K}\right)$ has a rigid cusp.

(iii) $S^{3} \backslash K$ non-normally covers some orbifold.

Notation In what follows, we shall let $Q_{K}=\mathbf{H}^{3} / C^{+}\left(\Gamma_{K}\right)$.

\subsection{Cusp fields and trace fields}

Recall that if $\Gamma$ is a Kleinian group of finite co-volume, the trace field is a finite extension of $\mathbb{Q}$. Furthermore, the invariant trace-field of $\Gamma, k \Gamma=\mathbb{Q}\left(\operatorname{tr}\left(\gamma^{2}\right): \gamma \in \Gamma\right)$, is a subfield of the trace-field that is an invariant of the commensurability class [13, Chapter 3]. When $\Gamma_{K}$ is the faithful discrete representation of $\pi_{1}\left(S^{3} \backslash K\right)$, it is shown in [19] that the invariant trace-field coincides with the trace-field. This also holds when the Kleinian group is generated by parabolic elements [20, Lemma 1].

For convenience we will often abuse the distinction between PSL and SL and simply work with matrices. If $\mathbf{H}^{3} / \Gamma$ is a 1-cusped hyperbolic 3-manifold, we can conjugate the peripheral subgroup to be

$$
\left\langle\left(\begin{array}{ll}
1 & 1 \\
0 & 1
\end{array}\right),\left(\begin{array}{ll}
1 & g \\
0 & 1
\end{array}\right)\right\rangle
$$

It is easily shown that $g \in k \Gamma$ (see for example [17, Proposition 2.7]) and $g$ is referred to as the cusp parameter of $\Gamma$. In the natural identification of the Teichmüller space of the torus with the upper half-plane, $g$ is the shape of the torus. The field $\mathbb{Q}(g)$ is called the cusp field, which is a subfield of $k \Gamma$.

Of relevance to us is that there are constraints on cusp parameters of tori that cover turnovers. The rigid orbifolds $S^{2}(2,4,4), S^{2}(2,3,6)$ and $S^{2}(3,3,3)$ have orbifold groups that are extensions of $\mathbb{Z} \oplus \mathbb{Z}$ by elements of orders 4,6 and 3 respectively. The maximal $\mathbb{Z} \oplus \mathbb{Z}$ subgroup in these cases can be conjugated to be:

$$
\left\langle\left(\begin{array}{ll}
1 & 1 \\
0 & 1
\end{array}\right),\left(\begin{array}{ll}
1 & \epsilon \\
0 & 1
\end{array}\right)\right\rangle
$$

Algebraic 83 Geometric Topology, Volume 8 (2008) 
where $\epsilon=i$ in the case of $S^{2}(2,4,4)$ and $\epsilon=(-1+\sqrt{-3}) / 2$ (which we shall denote by $\omega$ in what follows) otherwise. This discussion together with Proposition 2.1, yields the following corollary.

Corollary 2.2 Let $K$ be a hyperbolic knot which has hidden symmetries. Then the cusp parameter of $S^{3} \backslash K$ lies in $\mathbb{Q}(i)$ (when the turnover is $S^{2}(2,4,4)$ ) or in $\mathbb{Q}(\sqrt{-3})$ (when the turnover is $S^{2}(2,3,6)$ or $S^{2}(3,3,3)$ ).

\subsection{2-Bridge knots}

It will be convenient to recall some facts about 2-bridge knots that we shall make use of. In particular the work of Riley [21;22] is heavily used. Thus throughout this section let $K$ be a hyperbolic 2-bridge knot.

A 2-bridge knot $K$ has a normal form $(p, q)$ where $p$ and $q$ are odd integers and are determined by the lens space $L(p, q)$ that is the double cover of $S^{3}$ branched over $K$. The fundamental group of a 2-bridge knot complement has a presentation of the form $\pi_{1}\left(S^{3} \backslash K\right)=\left\langle x_{1}, x_{2}: r\right\rangle$ where $x_{1}$ and $x_{2}$ are meridians and the relation $r$ has the form $w x_{1} w^{-1}=x_{2}$ for some word $w$ in $x_{1}$ and $x_{2}$. The exponents of the $x_{i}$ in the word $w$ are all \pm 1 , and are determined by the 2-bridge normal form of $K$.

Let $\mathbf{F}$ be a field and fix an algebraic closure $\overline{\mathbf{F}}$. A homomorphism $\rho: \pi_{1}\left(S^{3} \backslash K\right) \rightarrow$ $\operatorname{PSL}(2, \mathbf{F})$ is called a parabolic representation (or simply $p$-rep for short) if $\rho\left(x_{1}\right)$ (and hence $\left.\rho\left(x_{2}\right)\right)$ is a parabolic element; ie conjugate in $\operatorname{PSL}(2, \overline{\mathbf{F}})$ to the element

$$
\left(\begin{array}{ll}
1 & 1 \\
0 & 1
\end{array}\right)
$$

If we conjugate so as to consider a p-rep normalized so that

$$
\rho\left(x_{1}\right)=\left(\begin{array}{ll}
1 & 1 \\
0 & 1
\end{array}\right) \text { and } \rho\left(x_{2}\right)=\left(\begin{array}{ll}
1 & 0 \\
y & 1
\end{array}\right),
$$

then Riley shows that $y$ satisfies a certain polynomial $\Lambda_{K}(y)$ with leading coefficient and constant term equal to 1 [21, Theorem 2]. We shall say that the above p-rep is in standard form.

In the case that $\mathbf{F}=\mathbb{C}, \Lambda_{K}$ is a polynomial with integral coefficients and some root of the p-rep polynomial corresponds to the faithful discrete representation of $\pi_{1}\left(S^{3} \backslash K\right)$ into PSL $(2, \mathbb{C})$. In this case, $\Lambda_{K}(y)$ is called the $p$-rep polynomial of $K$.

In [21], Riley also describes the image of a longitude $\ell$ for $x_{1}$ for a p-rep in standard form; namely a matrix

$$
\left(\begin{array}{ll}
1 & g \\
0 & 1
\end{array}\right)
$$

Algebraic 83 Geometric Topology, Volume 8 (2008) 
where $g=2 g_{0}$ and $g_{0}$ is an algebraic integer in the field $\mathbb{Q}(y)$.

Also important in what follows is Riley's work on p-reps when $\mathbf{F}$ has characteristic 2. Here the images of the meridians are elements of order 2, and thus the image of a p-rep is a dihedral group. This dihedral group is necessarily finite since a knot group cannot surject onto the infinite dihedral group. In addition, since the image groups are noncyclic, the dihedral groups considered are never of order 2 . Hence we exclude this case from further comment. Riley proves the following result which will be useful for us [21, Theorem 3].

Theorem 2.3 The p-rep polynomial $\Lambda_{K}(y)$ has no repeated factors modulo 2 , and so no repeated factors in $\mathbb{Z}[y]$.

This result allows us to prove Proposition 2.5 below (which Riley noticed in [22]). For completeness we give a proof. We first record the following standard facts about polynomials reduced modulo primes (for example see Koch [11, Proposition 3.8.1 and Theorem 3.8.2]).

Theorem 2.4 Let $f(x) \in \mathbb{Z}[x]$ be an irreducible monic polynomial, $\alpha$ a root and $k=\mathbb{Q}(\alpha)$ with ring of integers $R_{k}$. Let $d_{k}$ denote the discriminant of $k$, and $\Delta(\alpha)$ the discriminant of $f$. Let $p$ be a rational prime and $\bar{f}$ the reduction of $f$ modulo $p$. Then the following holds:

(i) $\bar{f}$ decomposes into distinct irreducible factors if and only if $p$ does not divide $\Delta(\alpha)$.

(ii) Suppose that $p$ does not divide $\Delta(\alpha) d_{k}^{-1}$ and $\bar{f}=\bar{f}_{1}^{e_{1}} \ldots \bar{f}_{g}^{e_{g}}$. Then $p R_{k}=$ $\mathcal{P}_{1}^{e_{1}} \ldots \mathcal{P}_{g}^{e_{g}}$ is the factorization into prime powers.

Proposition 2.5 Let $K$ be a hyperbolic 2-bridge knot with trace-field $k$. Then $\mathbb{Q}(i)$ is not a subfield of $k$.

Proof We shall show that 2 does not divide the discriminant of $k$. Since the discriminant of $\mathbb{Q}(i)$ is -4 , standard facts about the behavior of the discriminant in extensions of number fields precludes $\mathbb{Q}(i)$ from being a subfield of $k$ (see [11] for example).

Let $\rho$ denote the p-rep corresponding to the faithful discrete representation conjugated to be in standard form, and $\Lambda_{0}(y)$ be the irreducible factor of $\Lambda_{K}(y)$ which gives the representation corresponding to the complete structure. We denote the image group by $\Gamma_{K}$. Therefore $k=k \Gamma_{K}=\mathbb{Q}(y)$ for some root $y$ of $\Lambda_{0}(y)$. By Theorem 2.3 $\Lambda_{K}(y)$ has distinct factors modulo 2 , and so $\Lambda_{0}(y)$ has distinct factors modulo 2 . Thus, Theorem 2.4(i) shows that 2 does not divide the discriminant $\Delta(y)$ of $\Lambda_{0}(y)$. Since the discriminant of $\mathbb{Q}(y)$ divides $\Delta(y)$ (see [11, Chapter 3]) it follows that 2 does not divide the discriminant of $\mathbb{Q}(y)$. 
Proposition 2.5 shows that the cusp field of a hyperbolic 2-bridge knot is not $\mathbb{Q}(i)$. Hence if $K$ has hidden symmetries, Corollary 2.2 shows that the cusp of the orbifold $Q_{K}$ is either $S^{2}(3,3,3)$ or $S^{2}(2,3,6)$. In addition, notice that the element

$$
\mu=\left(\begin{array}{cc}
i & 0 \\
0 & -i
\end{array}\right)
$$

normalizes the p-rep $\rho$ in standard form. Hence $\mu \in N(K)<C^{+}\left(\Gamma_{K}\right)$, and we deduce:

Corollary 2.6 If $K$ is a hyperbolic 2-bridge knot with hidden symmetries the cusp of the orbifold $Q_{K}$ is $S^{2}(2,3,6)$ and the cusp field is $\mathbb{Q}(\sqrt{-3})$.

\subsection{Symmetry groups of 2-bridge knots}

The following discussion is well-known, but will be convenient for us to include.

We first state a result (originally due to Conway) about the symmetry groups of 2-bridge knot complements (a proof can be found in Sakuma [24] for example).

Notation Throughout the paper, we let $V$ denote the group $\mathbb{Z} / 2 \mathbb{Z} \oplus \mathbb{Z} / 2 \mathbb{Z}$ and $D_{n}$ the dihedral group with $2 n$ elements.

Theorem 2.7 Let $K$ be a hyperbolic 2-bridge knot. Then $\operatorname{Isom}^{+}\left(S^{3} \backslash K\right)=$ $N(K) / \Gamma_{K}$ is either $V$ or $D_{4}$. In both cases every nontrivial cyclic subgroup acts nonfreely (ie with nonempty fixed point set).

We need some additional information about the quotient orbifold $\mathbf{H}^{3} / N(K)$ when $K$ is 2 -bridge.

Lemma 2.8 Let $K$ be a hyperbolic 2-bridge knot and $Q_{K}=\mathbf{H}^{3} / N(K)$. Then:

(i) $N(K)$ is generated by elements of order 2 .

(ii) There is a unique 2-fold cover $Q^{\prime}=\mathbf{H}^{3} / \Gamma$ of $Q_{K}$ with a torus cusp. Furthermore, all torsion elements of $\Gamma^{\mathrm{ab}}=\Gamma /[\Gamma, \Gamma]=H_{1}\left(Q^{\prime}, \mathbb{Z}\right)$ have order 2 .

Proof We begin with a discussion of a particular subgroup (isomorphic to V) of the symmetry group of any hyperbolic 2-bridge knot complement. It is well-known that $\left(S^{3} \backslash K\right) / V$ is an orbifold whose orbifold group is generated by involutions. However, it will be convenient for what follows to recall a geometric description of this.

The complement of a 2-bridge knot in $S^{3}$ is the union of two three-balls $B_{1}$ and $B_{2}$ with two unknotted arcs deleted; see Figure 1. We take the two order two symmetries 

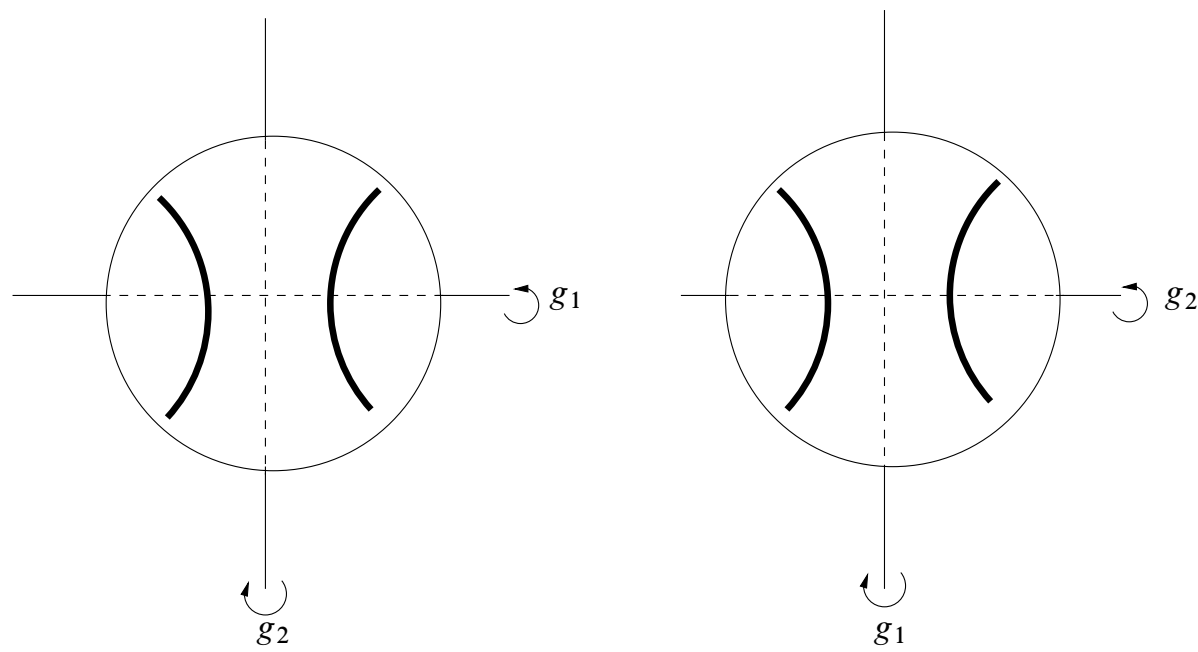

Figure 1: The action of $V$ on a 2-bridge knot complement

$g_{1}$ and $g_{2}$ which pointwise fix the centers of the arcs in $B_{1}$ and $B_{2}$ respectively to be the generators of $V$. Their composition is an order two isometry that fixes a circle which does not intersect the knot. Figure 1 below shows the axes of the symmetries $g_{1}$ and $g_{2}$. The axis of the order two symmetry $g_{1} g_{2}$ is perpendicular to the page.

By the solution to the Smith conjecture, the fixed point set of $g_{1} g_{2}$ in $S^{3}$ is an unknotted circle which does not intersect the knot. The quotient of $S^{3}$ by $g_{1} g_{2}$ is again $S^{3}$, and the image of the 2-bridge knot is another knot in $S^{3}$. We claim this knot is the unknot. Indeed, $g_{1} g_{2}$ is a symmetry whose fixed point set is disjoint from the knot and which takes one bridge to the other bridge. The fundamental group of the 2-bridge knot complement is generated by two elements $x_{1}$ and $x_{2}$. Fix a base point $b$ on the fixed point set of $g_{1} g_{2}$ in $B_{1}$. Then $x_{1}$ and $x_{2}$ can be represented by two curves which start at $b$ and encircle one of the two bridges in $B_{1}$. Now $g_{1} g_{2}$ acts on $\pi_{1}\left(S^{3} \backslash K\right)$ by setting $x_{1}=x_{2}$. Consider the orbifold fundamental group $\pi_{1}^{\text {orb }}(P)$ where $P$ is $S^{3} \backslash K$ modulo the action of $g_{1} g_{2} . \pi_{1}^{\text {orb }}(P)$ is obtained by adjoining an element $\beta$ to $\pi_{1}\left(S^{3} \backslash K\right)$ and adding the relations $\beta^{2}=1$ and $\beta x_{1} \beta=x_{2}$. The fundamental group of the underlying space of $P$ is the quotient of $\pi_{1}^{\text {orb }}(P)$ by the normal closure of $\beta$. Thus the fundamental group of the underlying space of $P$ is generated by one element. As above, this is the complement of a knot in $S^{3}$. Hence the knot is the unknot and the underlying space of $P$ is a solid torus. This proves the claim. The image of the singular set is also an unknot, and it wraps around the image of the knot. 
The fixed point sets of $g_{1}, g_{2}$ and $g_{1} g_{2}$ intersect in one point in each of the $B_{i}$. The orbifold $O=\left(S^{3} \backslash K\right) / V$ has the interior of a ball as its underlying space, and its cusp is a copy of $S^{2}(2,2,2,2)$ (Figure 2 is a schematic picture). The interior singular set of $O$ is a graph with two valence three vertices where all arcs have order two singularities. In Figure 2, the images of the fixed point sets of $g_{1}, g_{2}$ and $g_{1} g_{2}$ in $O$ are labeled by their respective group elements. We will refer to the image in $O$ of the fixed point set of $g_{1} g_{2}$ by $a_{g_{1}} g_{2}$. Note that $a_{g_{1} g_{2}}$ will in general wind around the arcs which meet the boundary. However, the arcs which meet the boundary are unknotted since unbranching along these arcs yields $P$, the quotient of the 2-bridge knot complement by $g_{1} g_{2}$, which has underlying space a solid torus.

Proof of (i) In the case that $\operatorname{Isom}^{+}\left(S^{3} \backslash K\right)=V, O=Q_{K}$. A presentation of the orbifold fundamental group $N(K)$ of $Q_{K}$ can be obtained by removing the singular set, taking a Wirtinger presentation of the complement, and then setting $\gamma^{2}=1$ for each generator. In this case, $N(K)$ is clearly generated by elements of order 2 .

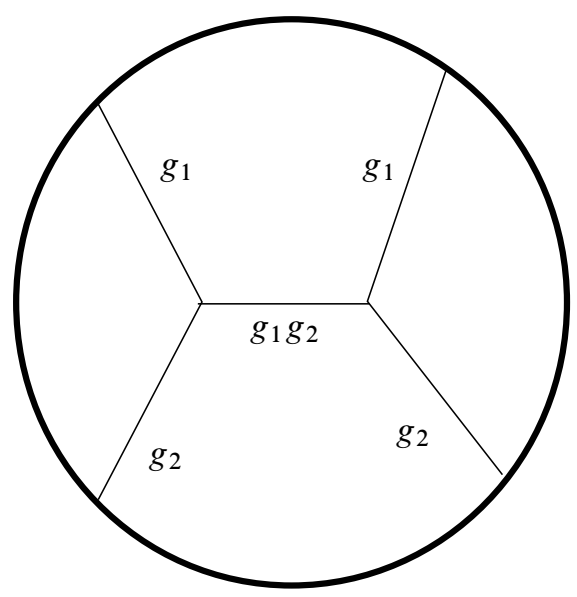

Figure 2: $O$, the 2-bridge knot complement modulo the action of $V$. The arcs of the singular set are order 2 .

In the case when $\operatorname{Isom}^{+}\left(S^{3} \backslash K\right)=D_{4}, Q_{K}=\mathbf{H}^{3} / N(K)$ is an orbifold which is double covered by $O$. Call the generator of the covering group $\tau$. Since the underlying space of $O$ is a ball, $\tau$ has fixed points acting on $O$. Denote the fixed point set of $\tau$ in $O$ by $b_{\tau}$. As $O$ is topologically a ball, $b_{\tau}$ is a properly embedded arc. This is unknotted by the solution to the Smith Conjecture.

The arc $b_{\tau}$ meets the cusp of $O$ in two points. Suppose one or both of these points coincide with the singular set of the cusp. Then there is an element of order 4 in $N(K)$ 
that fixes a point on the cusp. However, any isometry of a knot complement takes a longitude to a longitude. Hence if an isometry fixes a point on the cusp it can have order at most 2. Thus the fixed point set of $b_{\tau}$ is disjoint from the singular set of $O$ on the cusp at infinity.

Since $\tau$ takes the singular set of $O$, a finite tree, to itself, there is at least one fixed point in the interior of the singular set of $O$. We claim that there is exactly one, in the middle of $a_{g_{1} g_{2}}$. Since $\tau$ takes the singular set to itself, and $b_{\tau}$ does not intersect the singular set in the cusp, the only possible arc of intersection is $a_{g_{1}} g_{2}$. In this case $b_{\tau}$ is an arc from the cusp to the cusp which strictly contains $a_{g_{1} g_{2}}$. The preimage of $b_{\tau}$ in $S^{3} \backslash K$ is the fixed point set of some isometry $\tilde{\tau}$ of $S^{3} \backslash K$. This is either a circle or two arcs meeting the cusp. However, the preimage of $a_{g_{1} g_{2}}$ in $S^{3} \backslash K$ is a circle, and a circle or two arcs cannot properly contain a circle. Therefore $b_{\tau}$, the fixed point set of $\tau$, intersects the interior of the singular set of $O$ in points. Since $\tau$ takes the singular set to itself, any such point must be in the center of the arc $a_{g_{1}} g_{2}$, and there can only be one. This proves the claim. It follows that $Q_{K}=O /\langle\tau\rangle=\mathbf{H}^{3} / N(K)$ is an orbifold with a $S^{2}(2,2,2,2)$ cusp, and singular set consisting of a graph with order 2 arcs. Combinatorially, the graph of the singular set of $Q_{K}$ is a $H$, as is the graph of the singular set of $O$ in Figure 2. Again, the two arcs of the singular set which meet the boundary are unknotted. One of the arcs is the image of $b_{\tau}$ in $Q_{K}$ which is unknotted. The other arc is one unknotted arc of the singular set of $O$ identified to another. As before, using the Wirtinger presentation of the ball with the singular set removed, we see that $N(K)=\pi_{1}^{\text {orb }}\left(Q_{K}\right)$ can be generated by elements with order 2 . This proves (i).

Proof of (ii) As proved above, the cusp of $Q_{K}$ is a copy of $S^{2}(2,2,2,2)$. The only 2 -fold orbifold cover of this cusp which is a torus is obtained by unbranching along the four cone points. This is the kernel of a homomorphism $\phi: \pi_{1}^{\text {orb }}\left(S^{2}(2,2,2,2)\right) \rightarrow$ $\mathbb{Z} / 2 \mathbb{Z}=\{ \pm 1\}$ which sends each element of order two to -1 . To extend this cover to $Q_{K}$, we must unbranch along the curves of the singular set that meet the boundary. The singular set is combinatorially an $\mathrm{H}$, and the underlying space is topologically a ball. By using a Wirtinger presentation for the orbifold group, it follows easily that there can be no other unbranching. Therefore there is a unique 2-fold cover of $Q_{K}$ with a torus cusp. We denote this orbifold by $Q^{\prime}$. Since it was shown in the proof of (i) that the unbranching arcs are unknotted in $Q_{K}$, it follows that $Q^{\prime}$ has underlying space a solid torus. The singular set of the cover is the preimage of part of the singular set of $Q_{K}$, and so is of order 2. Let $\pi_{1}^{\text {orb }}\left(Q^{\prime}\right)$ denote the orbifold fundamental group of $Q^{\prime}$, and let $\psi: \pi_{1}^{\text {orb }}\left(Q^{\prime}\right) \rightarrow \mathbb{Z}$ be the homomorphism to the fundamental group of the underlying space of $Q^{\prime}$. 
If $\alpha$ is in the kernel of $\psi$, then $\alpha$ bounds an immersed disk in $X\left(Q^{\prime}\right)$, the underlying space of $Q^{\prime}$. Furthermore, this disc restricts to an immersed punctured sphere in $Q^{\prime} \backslash N\left(\Sigma\left(Q^{\prime}\right)\right)$, where $N\left(\Sigma\left(Q^{\prime}\right)\right)$ is a neighborhood of the ramification locus of $Q^{\prime}$. Therefore $\alpha=\sum a_{i}$ in $H_{1}\left(Q^{\prime}-N\left(\Sigma\left(Q^{\prime}\right)\right), \mathbb{Z}\right)$ and each $a_{i}$ is a meridian, ie, bounds a disk in $X\left(Q^{\prime}\right)$. Since $Q^{\prime}$ is obtained from $Q^{\prime}-N\left(\Sigma\left(Q^{\prime}\right)\right)$ by performing $(2,0)$ Dehn filling on the boundary components corresponding to $\Sigma\left(Q^{\prime}\right), 2 \alpha=\sum 2 a_{i}$ is trivial in $H_{1}\left(Q^{\prime}, \mathbb{Z}\right)=\Gamma^{\mathrm{ab}}$. Thus $[\alpha] \in H_{1}\left(Q^{\prime}, \mathbb{Z}\right)$ has order 2 when $\alpha$ is in the kernel of $\psi$. The map $\psi: \pi_{1}^{\text {orb }}\left(Q^{\prime}\right) \rightarrow \mathbb{Z}$ factors through the abelianization $H_{1}\left(Q^{\prime}, \mathbb{Z}\right) \rightarrow \mathbb{Z}$, so if $\alpha$ is not in the kernel of $\psi,[\alpha]$ has infinite order.

\section{Proof of Theorem 1.1}

The proof of Theorem 1.1 will follow immediately from the following two results.

Theorem 3.1 Let $K$ be a hyperbolic nonarithmetic 2-bridge knot. Then $K$ has no hidden symmetries.

Theorem 3.1 together with Proposition 2.1 shows that the minimal element in the orientable commensurability class of $\Gamma_{K}$ is the group $N(K)=N^{+}\left(\Gamma_{K}\right)$. Hence $Q_{K}=\mathbf{H}^{3} / N(K)$ and the proof of Theorem 1.1 is completed by:

Theorem 3.2 Let $Q_{K}$ be as above. Then $Q_{K}$ is covered by exactly one knot complement in $S^{3}$.

We defer the proof of Theorem 3.1 and the remainder of this section will be spent proving Theorem 3.2. For convenience we record the following result that will be used subsequently in several places. The nontrivial implication is Theorem 3.4 (1) in Gonzáles-Acuña and Whitten [7].

Theorem 3.3 Let $\Sigma$ be a homotopy 3-sphere and $K \subset \Sigma$ a nontrivial knot. Then $\Sigma \backslash K$ is nontrivially covered by the complement of a knot in a homotopy 3-sphere if and only if $\Sigma \backslash K$ admits a nontrivial cyclic surgery.

Of particular relevance to us is that in Takahashi [27] it is shown that a hyperbolic 2-bridge knot complement does not admit a nontrivial surgery with cyclic fundamental group. Therefore, we have:

Corollary 3.4 If $S^{3} \backslash K$ is a hyperbolic 2-bridge knot complement, it is not covered by the complement of any other knot in a homotopy 3 -sphere. 
Proof of Theorem 3.2 Throughout the proof of Theorem 3.2, $\Gamma_{K}$ will denote a faithful discrete $\mathrm{p}$-rep in standard form. Thus, the meridians $x_{1}$ and $x_{2}$ have images

$$
\left(\begin{array}{ll}
1 & 1 \\
0 & 1
\end{array}\right) \text { and }\left(\begin{array}{ll}
1 & 0 \\
y & 1
\end{array}\right)
$$

respectively.

Assume $K^{\prime}$ is a knot in $S^{3}$ such that $S^{3} \backslash K^{\prime}$ is commensurable with $S^{3} \backslash K$, and let $S^{3} \backslash K^{\prime}=\mathbf{H}^{3} / \Gamma_{K^{\prime}}$. We can assume that $\Gamma_{K^{\prime}}$ has been conjugated to be a subgroup of $N(K)$. Let $\Gamma=\left\langle\Gamma_{K}, \Gamma_{K^{\prime}}\right\rangle$ be the subgroup of $N(K)$ that is generated by $\Gamma_{K}$ and $\Gamma_{K^{\prime}}$, and let $\Delta=\Gamma_{K} \cap \Gamma_{K^{\prime}}$. We have the following lattice of subgroups of $\Gamma$. Note that Theorem 3.1 and Proposition 2.1 show that all the inclusions shown are of normal subgroups.

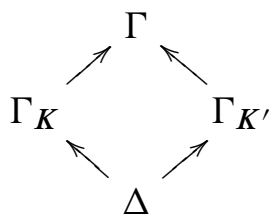

Now $\Gamma_{K^{\prime}} / \Delta \cong \Gamma / \Gamma_{K}$, which by Theorem 2.7, is a subgroup of $V$ or $D_{4}$. Since $\Gamma_{K^{\prime}}$ is a knot group, the quotient group $\Gamma_{K^{\prime}} / \Delta$ cannot be $V$ or $D_{4}$ (since both have noncyclic abelianization). Hence, the only possibilities for $\Gamma_{K^{\prime}} / \Delta \cong \Gamma / \Gamma_{K}$ are cyclic of order 1, 2 or 4 . Thus, since $\mathbf{H}^{3} / \Delta$ is a cyclic cover of $S^{3} \backslash K^{\prime}$, we deduce that $\mathbf{H}^{3} / \Delta$ has 1 torus cusp.

We claim that $\Gamma_{K} / \Delta$ is also cyclic. To see this, we have that $\mathbf{H}^{3} / \Delta \rightarrow S^{3} \backslash K$ is a regular cover by a 1-cusped manifold. Hence the peripheral subgroup of $\Gamma_{K}$ surjects onto the covering group. Therefore, the covering group is abelian, and hence cyclic since $K$ is a knot. Hence all of the inclusions indicated in the above diagram have cyclic quotients.

Note first that if $\Gamma / \Gamma_{K}$ is the trivial group then $\Gamma_{K^{\prime}}$ is a subgroup of $\Gamma_{K}$ which contradicts Corollary 3.4. Thus we assume henceforth that $\Gamma \neq \Gamma_{K}$.

Lemma 3.5 $\mathbf{H}^{3} / \Gamma$ is not a manifold but has a torus cusp.

Proof Since $\Gamma \neq \Gamma_{K}$, the last sentence in the statement of Theorem 2.7 immediately implies that $\mathbf{H}^{3} / \Gamma$ is an orbifold. Now suppose that $\mathbf{H}^{3} / \Gamma$ does not have a torus cusp. Since the cusp is the quotient of a torus, and not rigid (by Theorem 3.1 and Proposition $2.1)$, the cusp must be $S^{2}(2,2,2,2)$. This implies that the peripheral subgroup of $\Gamma$ fixing $\infty$ is

$$
\left\langle\left(\begin{array}{ll}
1 & 1 \\
0 & 1
\end{array}\right),\left(\begin{array}{ll}
1 & r \\
0 & 1
\end{array}\right),\left(\begin{array}{cc}
i & a \\
0 & -i
\end{array}\right)\right\rangle
$$


for some numbers $r$ and $a$, and where the third generator is the hyperelliptic involution of the torus that takes a peripheral element to its inverse.

As remarked in Section 2.2, since $\Gamma$ is generated by parabolic elements, we have that $\mathbb{Q}(\operatorname{tr} \Gamma)=k \Gamma=\mathbb{Q}(y)$. We claim that this is a contradiction.

To that end, consider the following products of elements in $\Gamma$;

$$
\left(\begin{array}{cc}
i & a \\
0 & -i
\end{array}\right)\left(\begin{array}{ll}
1 & 0 \\
y & 1
\end{array}\right) \text { and }\left(\begin{array}{cc}
i & a \\
0 & -i
\end{array}\right)\left(\begin{array}{ll}
1 & 0 \\
y & 1
\end{array}\right)\left(\begin{array}{ll}
1 & 1 \\
0 & 1
\end{array}\right) \text {. }
$$

The trace of the first product shows that $a \in \mathbb{Q}(y)$. The trace of the second product then shows that $i \in \mathbb{Q}(y)$, in contradiction to Proposition 2.5. This completes the proof of the lemma.

Lemma 3.6 $\Gamma / \Gamma_{K} \cong \Gamma / \Gamma_{K^{\prime}}$.

Proof The coverings $p_{1}: S^{3} \backslash K=\mathbf{H}^{3} / \Gamma_{K} \rightarrow \mathbf{H}^{3} / \Gamma$ and $p_{2}: S^{3} \backslash K^{\prime}=\mathbf{H}^{3} / \Gamma_{K^{\prime}} \rightarrow$ $\mathbf{H}^{3} / \Gamma$ are cyclic coverings of an orbifold with a torus cusp by a knot complement. Let $\tau_{1}$ and $\tau_{2}$ be the generators of the cyclic groups of covering transformations of the covers $p_{1}$ and $p_{2}$ respectively. The lemma will follow on establishing that each of $p_{1}$ and $p_{2}$ restricted to the cusp tori $T_{K}$ and $T_{K^{\prime}}$ of $S^{3} \backslash K$ and $S^{3} \backslash K^{\prime}$ respectively have the same degree. To that end, first observe that the preferred longitude

$$
\ell=\left(\begin{array}{cc}
1 & 2 g_{0} \\
0 & 1
\end{array}\right)
$$

of $S^{3} \backslash K$ is also a longitude for $S^{3} \backslash K^{\prime}$. To see this, we have already noted that both of these knot complements are cyclically covered by the 1-cusped manifold $\mathbf{H}^{3} / \Delta$. Since they are knot complements, their longitudes, and the nonseparating surfaces that these longitudes bound, both lift to this cyclic cover. Since there is only one homology class in the cusp of $\mathbf{H}^{3} / \Delta$ which bounds a nonseparating surface, it follows that $\ell$ is also a longitude of $S^{3} \backslash K^{\prime}$. By Lemma 3.5 the orbifold $\mathbf{H}^{3} / \Gamma$ has one torus cusp which we denote by $T_{\Gamma}$. Standard arguments in the orbifold setting (see the proof of Theorem 11 of Dunbar [6] for example) provide a nonseparating 2-orbifold in $\mathbf{H}^{3} / \Gamma$ which is bounded by a simple closed curve in $T_{\Gamma}$. We denote this class (which is unique) by $\ell_{0}$. The preimage of $\left[\ell_{0}\right] \in H_{1}\left(T_{\Gamma} ; \mathbb{Z}\right)$ must be $[\ell] \in H_{1}\left(T_{i} ; \mathbb{Z}\right)$ in both coverings. We deduce from these remarks that the covering degrees of $p_{1}$ and $p_{2}$ restricted to $\ell_{0}$ are the same.

The proof will be completed by establishing that $x_{1}$ and $x_{1}^{\prime}$, the meridians of $\Gamma_{K}$ and $\Gamma_{K}^{\prime}$, respectively, are primitive elements of $\Gamma$. Then the covering degree will be the degree restricted to $\ell_{0}$. To prove primitivity we argue as follows. Note that $\Gamma_{K}$ has 
algebraic integer entries. We first claim that $\Gamma$, and hence $\Gamma_{K^{\prime}}$, also has algebraic integer entries. Since $\Gamma$ has one torus cusp we can choose left coset representatives of $\Gamma_{K}$ in $\Gamma$ that are parabolic of the form

$$
\left(\begin{array}{ll}
1 & a \\
0 & 1
\end{array}\right)
$$

Since the property of having algebraic integer traces is a commensurability invariant,

$$
\operatorname{tr}\left(\begin{array}{ll}
1 & 0 \\
y & 1
\end{array}\right)\left(\begin{array}{ll}
1 & a \\
0 & 1
\end{array}\right)=2+a y
$$

is also an algebraic integer. Since $y$ is a unit $\left(\Lambda_{K}(y)\right.$ is monic with constant term 1$)$ $a$ is also an algebraic integer. Since this holds for any left coset representative, this proves the claim.

Now

$$
\begin{aligned}
\text { Now } & x_{1}=\left(\begin{array}{ll}
1 & 1 \\
0 & 1
\end{array}\right), \\
\text { and we shall assume that } \quad x_{1}^{\prime} & =\left(\begin{array}{ll}
1 & r \\
0 & 1
\end{array}\right) .
\end{aligned}
$$

Note that $r$ must be a unit, for if not, since we have shown all entries of $\Gamma_{K^{\prime}}$ are integral, we can find a prime ideal $\mathcal{P}$ dividing $\langle r\rangle$, and reducing the entries modulo $\mathcal{P}$ sends all of $\Gamma_{K^{\prime}}$ to the identity (since it is normally generated by $x_{1}^{\prime}$ ). However, this is impossible since there is an element in $\Gamma_{K^{\prime}}$ conjugating $x_{1}^{\prime}$ to a meridian $x_{2}^{\prime}$ fixing 0 . Such a conjugating matrix has zero as the $(1,1)$ entry.

If $x_{1}^{\prime}$ is not primitive, there is an element

such that

$$
\left(\begin{array}{ll}
1 & t \\
0 & 1
\end{array}\right) \in \Gamma
$$

$$
\left(\begin{array}{ll}
1 & t \\
0 & 1
\end{array}\right)^{n}=\left(\begin{array}{ll}
1 & r \\
0 & 1
\end{array}\right)
$$

for some integer $n$. We claim $n=1$. To see this, note that the elements of $\Gamma$ have algebraic integer entries by the argument above. Therefore $t=r / n$ is an algebraic integer. Since $r$ is a unit, $t= \pm r$, and $x_{1}^{\prime}$ is not a proper power of any element in $\Gamma$. It is clear that the same argument holds for $x_{1}$. Therefore, both $x_{1}$ and $x_{1}^{\prime}$ are primitive in $\Gamma$ as required.

From the previous discussion, we are assuming that $\Gamma / \Gamma_{K}=\Gamma / \Gamma_{K^{\prime}}$ is a cyclic group of order 2 or 4. 
In the case when $\Gamma / \Gamma_{K}=\Gamma / \Gamma_{K^{\prime}}=\mathbb{Z} / 4 \mathbb{Z}$ the theorem follows easily from Lemma 2.8. For then since $\mathbf{H}^{3} / \Gamma$ has a torus cusp, $\mathbf{H}^{3} / \Gamma$ is the unique 2 -fold cover $Q^{\prime}$ of $\mathbf{H}^{3} / N(K)$ with a torus cusp, and Lemma 2.8 shows that $\mathbf{H}^{3} / \Gamma$ has exactly one 4-fold cyclic cover. Therefore $\Gamma_{K}=\Gamma_{K^{\prime}}$.

We now assume that $\Gamma / \Gamma_{K}=\Gamma / \Gamma_{K^{\prime}}=\mathbb{Z} / 2 \mathbb{Z}$. In this case all the cyclic quotients arising from the lattice of subgroups at the beginning of the proof of Theorem 3.2 are order 2. In particular, since $K$ is $2-$ bridge, $\mathbf{H}^{3} / \Delta$ is the complement of a knot in a lens space.

Recall the proof of Lemma 3.6 shows that $x_{1}$ and $x_{1}^{\prime}$ are primitive elements in $\Gamma$. Furthermore, $x_{1} \notin \Gamma_{K}^{\prime}$ and $x_{1}^{\prime} \notin \Gamma_{K}$. For if so, then $x_{1} \in \Gamma_{K} \cap \Gamma_{K^{\prime}}=\Delta$ which is false. If $x_{1}^{\prime} \in \Gamma_{K}$ then $x_{1}^{\prime} \in \Delta$ and normality implies that $\Delta=\Gamma_{K^{\prime}}$ which is false as $\left[\Gamma_{K^{\prime}}: \Delta\right]=2$. Consider the normal closure of $x_{1}^{2}$ in $\Gamma$. We denote this by $\left\langle x_{1}^{2}\right\rangle_{\Gamma}$. We claim that $\left\langle x_{1}^{2}\right\rangle_{\Gamma}=\left\langle x_{1}^{2}\right\rangle_{\Gamma_{K}}$. The inclusion $\left\langle x_{1}^{2}\right\rangle_{\Gamma_{K}} \subset\left\langle x_{1}^{2}\right\rangle_{\Gamma}$ is clear. For the reverse inclusion, we can choose $x_{1}^{\prime}$ to be a left coset representative for $\Gamma_{K}$ in $\Gamma$ and it follows that $\Gamma=\left\langle\Gamma_{K}, x_{1}^{\prime}\right\rangle$. Since $x_{1}^{\prime}$ commutes with $x_{1}^{2}$, we deduce that $\left\langle x_{1}^{2}\right\rangle_{\Gamma} \subset\left\langle x_{1}^{2}\right\rangle_{\Gamma_{K}}$.

The exact same argument with $\Gamma_{K}$ and $x_{1}^{\prime}$ replaced by $\Gamma_{K}^{\prime}$ and $x_{1}$ shows that $\left\langle x_{1}^{2}\right\rangle_{\Gamma}=$ $\left\langle x_{1}^{2}\right\rangle_{\Gamma_{K}^{\prime}}$. Similarly, we can take $x_{1}$ to be a left coset representative of $\Delta$ in $\Gamma_{K}$, which also shows that $\left\langle x_{1}^{2}\right\rangle_{\Delta}=\left\langle x_{1}^{2}\right\rangle_{\Gamma_{K}}$. Thus we conclude that $\left\langle x_{1}^{2}\right\rangle_{\Gamma_{K}}=\left\langle x_{1}^{2}\right\rangle_{\Gamma_{K}^{\prime}}=\left\langle x_{1}^{2}\right\rangle_{\Delta}$. For convenience we denote $\left\langle x_{1}^{2}\right\rangle_{\Gamma}$ by $\mathcal{N}$.

Now the group $\Gamma_{K} / \mathcal{N}$ is the orbifold fundamental group of the orbifold obtained by the $(2,0)$ Dehn filling on the 2-bridge knot $K$. Now the double branched cover of $K$ is a lens space $L(p / q)$ whose fundamental group is the cyclic group of (odd) order $p$. Since this double cover is obtained by first performing $(2,0)$ orbifold Dehn surgery on $K$, and then passing to the index 2 cover which is a manifold, we deduce that $\Gamma_{K} / \mathcal{N}$ is a dihedral group of order $2 p$ for some odd integer $p$.

Note that $x_{1}^{2}$ is a primitive element of $\Gamma_{K^{\prime}}$. For if not, then $x_{1} \in \Gamma_{K^{\prime}}$, but as noted above $x_{1} \notin \Delta$. Therefore, $\Gamma_{K}^{\prime} / \mathcal{N}$ and $\Delta / \mathcal{N}$ are the fundamental groups of the manifolds obtained by Dehn filling the primitive curve $x_{1}^{2}$ in $S^{3} \backslash K^{\prime}$ and $\mathbf{H}^{3} / \Delta$ respectively. In particular, this latter group is a cyclic group of order $p$ (denoted $C_{p}$ ). We denote the former group by $G^{\prime}$. Hence we have the following diagram of groups:

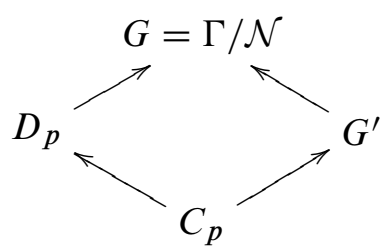

Algebraic 83 Geometric Topology, Volume 8 (2008) 
The group $G$ is a 2 -fold extension of $D_{p}$, and so has order $4 p$. The group $G^{\prime}$ is a finite group of order $2 p$ arising as the fundamental group of a closed orientable 3 -manifold. We claim that the finite group $G^{\prime}$ is cyclic.

This will complete the proof of Theorem 3.2. For if $G^{\prime}$ is cyclic, then $S^{3} \backslash K^{\prime}$ has a $2 p$-fold cyclic cover $\Sigma \backslash K^{\prime \prime}$, where $\Sigma$ is a homotopy 3 -sphere. Note that since $\Sigma \backslash K^{\prime \prime}$ is the $2 p$-fold cyclic cover of $S^{3} \backslash K^{\prime}$, it must cover the 2-fold cover of $S^{3} \backslash K^{\prime}$, namely $\mathbf{H}^{3} / \Delta$. Hence $\Sigma \backslash K^{\prime \prime}$ covers $S^{3} \backslash K$ which contradicts Corollary 3.4.

To establish that $G^{\prime}$ is cyclic, by Milnor [15] there are a limited number of types of noncyclic finite groups that can be the fundamental group of a closed orientable 3-manifold. We list these below using the notation of Boyer and Zhang [4]:

- Even D-type: $\left\{x, y: x^{2}=(x y)^{2}=y^{n}\right\} \times \mathbb{Z} / j \mathbb{Z}$, with $j \geq 1, n \geq 2$ with $n$ even. The abelianization is $\mathbb{Z} / 2 \mathbb{Z} \oplus \mathbb{Z} / 2 j \mathbb{Z}$.

- Odd D-type: $\left\{x, y: x^{2^{k}}=1, y^{2 l+1}=1, x y x^{-1}=y^{-1}\right\} \times \mathbb{Z} / j \mathbb{Z}$, with $j \geq 1$, $(2(2 l+1), j)=1 \quad k \geq 2$. The abelianization is $\mathbb{Z} / 2^{k} j \mathbb{Z}$.

- T-type: $\left\{x, y, z: x^{2}=(x y)^{2}=y^{2}, z^{3^{k}}=1, z x z^{-1}=y, z y z^{-1}=x y\right\} \times$ $\mathbb{Z} / j \mathbb{Z},(6, j)=1$. The abelianization is $\mathbb{Z} / 3^{k} j \mathbb{Z}$.

- O-type: $\left\{x, y: x^{2}=(x y)^{3}=y^{4}, x^{4}=1\right\} \times \mathbb{Z} / j \mathbb{Z},(6, j)=1$. The abelianization is $\mathbb{Z} / 2 j \mathbb{Z}$.

- I-type: $\left\{x, y: x^{2}=(x y)^{4}=y^{5}, x^{4}=1\right\} \times \mathbb{Z} / j \mathbb{Z},(30, j)=1$. The abelianization is $\mathbb{Z} / j \mathbb{Z}$.

- Q-type: $\left\{x, y, z: x^{2}=(x y)^{2}=y^{2 n}, z^{k l}=1, x z x^{-1}=z^{r}, y z y^{-1}=z^{-1}\right\} \times$ $\mathbb{Z} / j \mathbb{Z}, n, k, l, j$ relatively prime odd positive integers, $r \equiv-1 \bmod k, r \equiv 1$ $\bmod l$. The abelianization is $\mathbb{Z} / 2 \mathbb{Z} \oplus \mathbb{Z} / 2 j \mathbb{Z}$.

Now $\left|G^{\prime}\right|=2 p$ where $p$ is odd. This allows us to immediately rule out both $D$-types, $O$-type, $I$-type and $Q$-type, since either the fundamental group or its abelianization is clearly divisible by 4 . Since $G^{\prime}$ has order $2 p$ and contains a cyclic subgroup of order $p$, $G^{\prime}$ surjects onto $\mathbb{Z} / 2 \mathbb{Z}$. This precludes $G^{\prime}$ being of $T$-type, since the abelianization of a group of $T$-type is odd. This proves that $G^{\prime}$ is a cyclic group as claimed.

\section{2-Bridge knots and hidden symmetries}

In this section we prove Theorem 3.1. This will be done in Section 4.1 and Section 4.2. We begin with some preliminary discussion. 


\subsection{Preliminaries to prove Theorem 3.1}

$K$ is a hyperbolic 2-bridge knot different from the figure-eight knot, and as above, $\Gamma_{K}$ the faithful discrete p-rep of $\pi_{1}\left(S^{3} \backslash K\right)$ in standard form; ie given by

$$
\rho\left(x_{1}\right)=\left(\begin{array}{ll}
1 & 1 \\
0 & 1
\end{array}\right) \text { and } \rho\left(x_{2}\right)=\left(\begin{array}{ll}
1 & 0 \\
y & 1
\end{array}\right) \text {. }
$$

As above the invariant trace-field is $\mathbb{Q}(y)$, and we let the ring of integers of $\mathbb{Q}(y)$ be denoted by $R_{y}$. Assuming that $K$ has hidden symmetries, Corollary 2.6 shows that the orientable commensurator orbifold $Q_{K}$ has a $S^{2}(2,3,6)$ rigid cusp. Hence $\mathbb{Z}[\omega] \subset R_{y}$.

Let $\wp \subset R_{y}$ be a prime ideal that divides the principal ideal $2 R_{y} \subset R_{y}$, and let $\mathbf{F}=R_{y} / \wp$. This is a finite field of order $2^{s}$ for some integer $s \geq 1$. In fact, since $\mathbb{Z}[\omega] \subset R_{y}$ and 2 is inert in $\mathbb{Z}[\omega]$, it follows that $|\mathbf{F}|=4^{s}$ for some integer $s \geq 1$. Let $\phi: \operatorname{PSL}\left(2, R_{y}\right) \rightarrow \operatorname{PSL}(2, \mathbf{F})$ be the reduction homomorphism. The key result that is needed to prove Theorem 3.1 is the following.

Theorem 4.1 The image of $\Gamma_{K}$ under $\phi$ is a dihedral group of order 6 or 10 .

Deferring the proof of Theorem 4.1 until Section 4.2, we complete the proof of Theorem 3.1 .

As in Section 2.3 we let $\Lambda_{0}(y)$ denote the factor of the p-rep polynomial $\Lambda_{K}(y)$ that corresponds to the complete hyperbolic structure. Let $\bar{\Lambda}_{0}(y)$ be the reduction of $\Lambda_{0}(y)$ modulo 2. By Theorem 2.3, $\bar{\Lambda}_{0}(y)$ has no repeated factors, and each factor corresponds to a dihedral representation of $\Gamma_{K}$. By Theorem 4.1 the image of these representations are dihedral of order 6 or 10. Now from [21, Proposition 4], we deduce that the corresponding factors of $\bar{\Lambda}_{0}(y)$ have degrees 1 and 2 respectively. Since we are working modulo 2 , the only possible irreducible polynomials are $x$ in the case of $D_{3}$ and $x^{2}+x+1$ in the case of $D_{5}$.

By Theorem 2.3 and Theorem 2.4(i) 2 does not divide the discriminant $\Delta(y)$ of $\Lambda_{0}(y)$. Hence 2 does not divide $\Delta(y) d_{k}^{-1}$, where $d_{k}$ is the discriminant of $\mathbb{Q}(y)$. Therefore Theorem 2.4(ii) shows that the above factors determine the decomposition of the principal ideal $2 R_{y}$ into prime (ideal) power factors. Any such $\wp$ as above is one of these factors. Since there are no repeated factors, we deduce that the decomposition of $2 R_{y}$ is into at most the product of two prime ideals of $R_{y}$. Therefore, $\bar{\Lambda}_{0}(y)$ has degree at most 3 . Hence $\Lambda_{0}(y)$ has degree at most 3 (recall $\Lambda_{0}(y)$ is a monic polynomial so the leading coefficient is never zero modulo 2 ). 
The degree cannot be 1 , since $y$ is a nonreal root of the p-rep polynomial. If the degree is 2 , then since $\mathbb{Q}(y)$ contains $\mathbb{Q}(\sqrt{-3})$ we have that $\mathbb{Q}(y)=\mathbb{Q}(\sqrt{-3})$ and so $\Gamma_{K}$ has traces in $\mathbb{Z}[\omega]$. It follows that $\Gamma_{K}$ is arithmetic ( $\operatorname{cf}[20]$ ) which is false. Finally, the degree cannot be 3 , because $\mathbb{Q}(\sqrt{-3}) \subset \mathbb{Q}(y)$. This contradiction completes the proof.

\subsection{Proof of Theorem 4.1}

Throughout this subsection we let $C(K)=C^{+}\left(\Gamma_{K}\right)$ which is assumed to contain $\Gamma_{K}$. Since $\Gamma_{K}$ contains a peripheral subgroup fixing $\infty, C(K)$ has a peripheral subgroup fixing $\infty$ and we denote this by $B$ (so $B$ is isomorphic to the orbifold group of $\left.S^{2}(2,3,6)\right)$. We begin with some preliminary lemmas.

Lemma 4.2 The orbifold group $B<C(K)$ has the form

$$
\left\langle\left(\begin{array}{ll}
1 & 1 \\
0 & 1
\end{array}\right),\left(\begin{array}{ll}
1 & \omega \\
0 & 1
\end{array}\right),\left(\begin{array}{cc}
i \omega & 0 \\
0 & -i \bar{\omega}
\end{array}\right)\right\rangle
$$

Proof $B$ fixes infinity and so an element of order 6 in $B$ has the form

$$
e=\left(\begin{array}{cc}
i \omega & t \\
0 & -i \bar{\omega}
\end{array}\right)
$$

for some number $t$. We claim that we can arrange that $t$ can be taken to be 0 .

To see this we argue as follows. By [17, Theorem 2.2], there is a normal subgroup $L$ of $C(K)$ (with quotient $(\mathbb{Z} / 2 \mathbb{Z})^{\alpha}$ ) such that all elements of $C(K)$ whose trace lies in $\mathbb{Q}(y) \backslash\{0\}$ are elements of $L$. Recall from the proof of Proposition 2.5 the element $\mu \in N(K)<C(K)$. Hence the element

$$
\delta=e \mu=\left(\begin{array}{cc}
-\omega & -t i \\
0 & -\bar{\omega}
\end{array}\right)
$$

is an element of order 3 which lies in $L$. Now $L$ also contains $\Gamma_{K}$ and so,

$$
\operatorname{tr}\left(\rho\left(x_{2}\right) \delta\right)=-\omega-\bar{\omega}-y t i \in \mathbb{Q}(y) .
$$

Furthermore, since traces of elements in $\Gamma_{K}$ are algebraic integers, all traces of elements in $C(K)$ are algebraic integers by commensurability. Letting $R_{y}$ denote the ring of integers in $\mathbb{Q}(y)$ we deduce that $y t i \in R_{y}$. Since $y$ is a unit we deduce that $t i \in R_{y}$.

Now

$$
e^{3}=\left(\begin{array}{cc}
-i & 2 t \\
0 & i
\end{array}\right)
$$


is an element of order 2 in $B$, and the product

$$
\mu e^{3}=\left(\begin{array}{cc}
1 & 2 t i \\
0 & 1
\end{array}\right)
$$

is a parabolic element in $B$. Since the cusp field is $\mathbb{Q}(\sqrt{-3})$ it follows that $t i \in$ $\mathbb{Q}(\sqrt{-3})$. Furthermore from above $t i$ is an algebraic integer, and so $t i \in \mathbb{Z}[\omega]$. Hence the element $\delta$ above has coefficients in $\mathbb{Z}[\omega]$. Let $x=-t i \bar{\omega} \in \mathbb{Z}[\omega]$, and consider the product

$$
\delta\left(\begin{array}{ll}
1 & x \\
0 & 1
\end{array}\right)
$$

This gives the element $\quad\left(\begin{array}{cc}\omega & 0 \\ 0 & \bar{\omega}\end{array}\right)$.

Taking the product with $\mu$ gives the desired element of order 6 .

Lemma 4.3 $C(K)$ contains a subgroup $C_{0}(K)$ of index 2 such that $\Gamma_{K}$ is a subgroup of $C_{0}(K)$ and $\mathbf{H}^{3} / C_{0}(K)$ has a $S^{2}(3,3,3)$ cusp. Furthermore, $C_{0}(K)<\operatorname{PSL}\left(2, R_{y}\right)$.

Proof As mentioned in the proof of Lemma 4.2, Theorem 2.2 of [17] provides a normal subgroup $L$ of $C(K)$ with quotient $(\mathbb{Z} / 2 \mathbb{Z})^{\alpha}$ such that all elements of $C(K)$ whose trace lies in $\mathbb{Q}(y) \backslash\{0\}$ are elements of $L$. As noted, $L$ contains $\Gamma_{K}$, and so $\mathbf{H}^{3} / L$ has one cusp. Since $\mathbf{H}^{3} / L$ has one cusp, $B$ must surject onto the covering group $(\mathbb{Z} / 2 \mathbb{Z})^{\alpha}$. We claim that this forces $\alpha=1$.

To see this, note that the abelianization of the group $B$ is $\mathbb{Z} / 6 \mathbb{Z}$. Hence, the image of $B$ under the homomorphism $C(K) \rightarrow C(K) / L$ is cyclic, and so $\alpha \leq 1$. However, $\alpha \neq 0$ since an element of order 6 in $B$ cannot lie in $L$. For if so, then $\sqrt{3} \in \mathbb{Q}(y)$. Since $\mathbb{Q}(\sqrt{-3}) \subset \mathbb{Q}(y)$ (by Corollary 2.6), it follows that $\sqrt{3} \sqrt{-3}=3 i \in \mathbb{Q}(y)$ which contradicts Proposition 2.5. Given that the element

$$
\left(\begin{array}{cc}
\omega & 0 \\
0 & \bar{\omega}
\end{array}\right)
$$

constructed in the proof of Lemma 4.2 is an element of $L$, this argument also shows that the cusp of $\mathbf{H}^{3} / L$ is $S^{2}(3,3,3)$.

Note that $L<\operatorname{PSL}\left(2, R_{y}\right)$. For since $\mathbf{H}^{3} / L$ has one cusp, a system of coset representatives of $\Gamma_{K}$ in $L$ can be taken from the cusp subgroup $E$ of $L$. By definition of $B$, the subgroup $E$ is:

$$
\left\langle\left(\begin{array}{ll}
1 & 1 \\
0 & 1
\end{array}\right),\left(\begin{array}{ll}
1 & \omega \\
0 & 1
\end{array}\right),\left(\begin{array}{cc}
\omega & 0 \\
0 & \bar{\omega}
\end{array}\right)\right\rangle
$$


Thus all the coefficients lie in $\mathbb{Z}[\omega]$. Now Corollary 2.6 implies that $\mathbb{Z}[\omega] \subset R_{y}$. Hence all the coefficients of $L$ are elements of $R_{y}$. We now take $C_{0}(K)=L$.

Consider the reduction homomorphism $\phi$ restricted to the group $C_{0}(K)$ (which is a subgroup of PSL $\left(2, R_{y}\right)$ by Lemma 4.3). We continue to denote this by $\phi$ and let $\Delta=\operatorname{ker} \phi$. As in the proof of Lemma 4.3,

$$
E=\left\langle\left(\begin{array}{ll}
1 & 1 \\
0 & 1
\end{array}\right),\left(\begin{array}{cc}
1 & \omega \\
0 & 1
\end{array}\right),\left(\begin{array}{cc}
\omega & 0 \\
0 & \bar{\omega}
\end{array}\right)\right\rangle
$$

denotes the cusp subgroup of the $C_{0}(K)$. Note that $E \cap \Delta$ is torsion free, since the element

$$
\left(\begin{array}{cc}
\omega & 0 \\
0 & \bar{\omega}
\end{array}\right)
$$

of order 3 injects under $\phi$.

We claim that $\phi(E)$ has order 12 . Indeed, since $E<\operatorname{PSL}(2, \mathbb{Z}[\omega])<\operatorname{PSL}\left(2, R_{y}\right)$ it suffices to consider the image of $E$ under the reduction homomorphism restricted to $\operatorname{PSL}(2, \mathbb{Z}[\omega])$. By the definition of $E$ and $\phi$ it is easily seen that $\phi(E)$ is an extension of $V$ by $\mathbb{Z} / 3 \mathbb{Z}$. This defines a subgroup of order 12 , which proves the claim.

Now $\Gamma_{K} \cap \Delta$ is the kernel of $\phi$ restricted to $\Gamma_{K}$. As discussed in Section 2.3 this is a dihedral representation. We need to show that $\Gamma_{K} / \Gamma_{K} \cap \Delta$ is a dihedral group of order 6 or 10.

Lemma 4.4 $\Gamma_{K} / \Gamma_{K} \cap \Delta$ is a dihedral group of order $2 m$ and $m$ is odd.

Proof That $\Gamma_{K} \cap \Delta$ is dihedral follows from the sentence before the lemma. Since $K$ is a 2-bridge knot it has a 2-bridge normal form as discussed in Section 2.3, and hence the double branched cover of $K$ is a lens space $L(p / q)$ whose fundamental group is the cyclic group of odd order $p$.

The double cover of a manifold branched over a knot $K$ can be obtained by first performing $(2,0)$ orbifold Dehn surgery on $K$, and then passing to the index 2 cover which is a manifold. In particular meridians of $K$ are mapped to elements of order 2, and furthermore any quotient of $\pi_{1}\left(S^{3} \backslash K\right)$ in which the meridians are mapped to elements of order 2 is a quotient of the orbifold group obtained above.

Hence, in the case at hand, the orbifold group is a dihedral group of order $2 p$ where $p>1$ is odd. The lemma now follows from the discussion in the previous paragraph. 
We now complete the proof of Theorem 4.1. As before, if $\ell$ denotes the longitude for $x_{1}$ described in Section 2.3, then

$$
\rho(\ell)=\left(\begin{array}{ll}
1 & g \\
0 & 1
\end{array}\right)
$$

where $g=2 g_{0}$ and $g_{0} \in R_{y}$. Hence $\phi(\rho(\ell))=1$, and so the image of the peripheral subgroup $\left\langle\rho\left(x_{1}\right), \rho(\ell)\right\rangle$ under $\phi$ is cyclic of order 2 . Hence the cover of $S^{3} \backslash K$ determined by $\Gamma_{K} \cap \Delta$ has $m$ cusps (and $m$ is odd by Lemma 4.4).

We now count the cusps of $\mathbf{H}^{3} /\left(\Gamma_{K} \cap \Delta\right)$ in a different way. Consider the following diagram of subgroups of $C_{0}(K)$ :

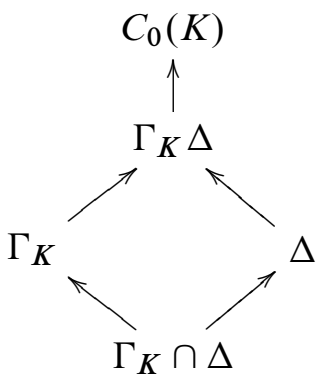

Since $\Gamma_{K} \Delta$ contains $\Gamma_{K}$ and is contained in $C_{0}(K)$, it has one cusp which is either a torus or $S^{2}(3,3,3)$. Furthermore, $\Gamma_{K} \Delta / \Delta \cong \Gamma_{K} /\left(\Gamma_{K} \cap \Delta\right)=D_{m}$. We claim that the cusp of $\mathbf{H}^{3} / \Gamma_{K} \Delta$ is a torus. To see this we argue as follows. Assume that the cusp is $S^{2}(3,3,3)$. Denote the cusp subgroup of $\Gamma_{K} \Delta$ fixing $\infty$ by $E^{\prime}$. Arguing as above for $\phi(E)$ shows that $\phi\left(E^{\prime}\right)$ has order 12 . Since $\phi\left(\Gamma_{K} \Delta\right)=D_{m}, 12$ must divide $2 m$. However, this contradicts Lemma 4.4 which shows $m$ is odd.

Therefore the cusp of $\Gamma_{K} \Delta$ is a torus, and we have a covering space $S^{3} \backslash K \rightarrow$ $\mathbf{H}^{3} / \Gamma_{K} \Delta$. By [20, Lemma 4], this is a regular abelian cover. By Theorem 2.7 it follows that the covering group is cyclic of order 1,2 or 4 or it is the group $V$.

The image of $C_{0}(K)$ under $\phi$ is a subgroup of $\operatorname{PSL}(2, \mathbf{F})$, where as discussed in Section 4.1, $|\mathbf{F}|=4^{s}=q$. By [26, Theorem 6.25], subgroups of $\operatorname{PSL}(2, \mathbf{F})$ are as follows:

(1) $\operatorname{PSL}\left(2, \mathbf{F}^{\prime}\right)$ where $\mathbf{F}^{\prime}$ is a subfield of $\mathbf{F}$ of order $2^{k}$. Note that since the characteristic is $2, \operatorname{PGL}\left(2, \mathbf{F}^{\prime}\right)=\operatorname{PSL}\left(2, \mathbf{F}^{\prime}\right)$ which excludes one of the possibilities of [26, Theorem 6.25].

(2) $A_{4}$ or $A_{5}$. Note that $S_{4}$ is ruled out by [26, Theorem 6.26(C)].

(3) A subgroup $H$ of order $q(q-1)$ and its subgroups. A Sylow 2-subgroup $Q<H$ is elementary abelian and $H / Q$ is cyclic of order $q-1$. 
(4) A dihedral group of order $2(q \pm 1)$ or one of its subgroups.

We handle each possibility in turn.

Case 1 Assume that $C_{0}(K)$ maps onto $\operatorname{PSL}\left(2, \mathbf{F}^{\prime}\right)$. From above we know that $\left[\Gamma_{K} \Delta\right.$ : $\left.\Gamma_{K}\right]=1,2,4$. If $\left[\Gamma_{K} \Delta: \Gamma_{K}\right]=1$ then $\Delta=\Gamma_{K} \cap \Delta$. Now the number of cusps of $\mathbf{H}^{3} / \Delta$ is given by $\left|\operatorname{PSL}\left(2, \mathbf{F}^{\prime}\right)\right| /|\phi(E)|$, which is $2^{k}\left(2^{2 k}-1\right) / 12$ (by our computation of $|\phi(E)|$ given above ). From above, the number of cusps is the odd integer $m$. Thus $k=2$, from which it follows that $m=5$, thus yielding the dihedral group $D_{5}$.

In the case that $\Gamma_{K} \Delta / \Gamma_{K}$ is cyclic of order 2 or 4 or $V$, we have $\Delta /\left(\Gamma_{K} \cap \Delta\right)$ is cyclic of order 2 or 4 or $V$. Thus we count that $\mathbf{H}^{3} / \Delta$ can have $m, m / 2$, or $m / 4$ cusps. Since $m$ is odd, $\Delta$ has $m$ cusps. We now argue as above, and we deduce that $k=2$ and $m=5$ yielding the dihedral group $D_{5}$ again.

Case 2 Assume that $C_{0}(K)$ maps onto $A_{4}$ or $A_{5}$. The only noncyclic dihedral subgroup of $A_{4}$ is $V$ and $\Gamma_{K}$ cannot map onto this since it is a knot group. The only noncyclic dihedral subgroups of $A_{5}$ are $D_{5}$ and $D_{3}$. Hence we are done in this case.

Case 3 Suppose that the image of $C_{0}(K)$ is a subgroup of $H$ as given above. $H$ contains the image of $\Gamma_{K}$. This is a dihedral subgroup $D_{m}$ of order $2 m$, where $m$ is odd. Since $Q$ is normal in $H, Q \cap D_{m}$ is normal in $D_{m}$, and the only normal subgroup of $D_{m}$ is the cyclic subgroup of order $m$. Since $Q$ is a 2-group, 2 must divide $m$, but $m$ is odd.

Case 4 Suppose that the image of $C_{0}(K)$ is a subgroup of a dihedral group of order $2(q \pm 1)$. The order of the image of $E$ is 12 . Hence 12 divides $2\left(4^{s} \pm 1\right)$, which is absurd. This completes the proof.

\section{Commensurable knot complements}

\subsection{Commensurability classes containing more than one knot complement}

In the nonhyperbolic case, infinitely many knot complements can easily occur in one commensurability class. For example, torus knots, in particular nonhyperbolic 2-bridge knots, all have commensurable complements (see Neumann [16]).

As mentioned in Section 1, there are examples of hyperbolic knots $K$ for which the commensurability class of $S^{3} \backslash K$ contains more than one knot complement. We now describe the constructions that are known to us. 
Lens space surgeries The main source of examples occurs when $K$ admits a lens space surgery. In this case $S^{3} \backslash K$ has a cyclic cover that is a knot complement by Theorem 3.3. By [5], there can be at most two nontrivial cyclic surgeries. Hence if a hyperbolic knot $K$ admits a lens space surgery there can be up to 3 knot complements in the commensurability class that arise from this construction. This holds for the $(-2,3,7)$-pretzel knot complement (see Berge [2]).

Hidden symmetries A pair of commensurable knot complements that do not arise as above are the two dodecahedral knot complements of Aitchison and Rubinstein [1]. These are commensurable of the same volume, and have hidden symmetries (see Neumann and Reid [17]).

If a hyperbolic knot $K$ has no hidden symmetries then there are only finitely many knot complements in the commensurability class of $S^{3} \backslash K$ (see [20, Theorem 5]). However, in the presence of hidden symmetries, it is unknown to the authors whether there are finitely many knot complements in a commensurability class (even for the dodecahedral knots).

Symmetries The final construction we are aware of was described to us by Weumann (personal communication). He has constructed an infinite family of pairs of knots $\left\{K_{i}, K_{i}^{\prime}\right\}$ which have the following property. The complements $S^{3} \backslash K_{i}$ and $S^{3} \backslash K_{i}^{\prime}$ have different volumes, and are both regular covers of a common (genuine) orbifold.

The simplest pair of examples are the knots $9_{48}$ and $12 n 642$, where the volume ratio is $4: 3$. See Goodman, Heard and Hodgson [8] for a description of the common orbifold that they cover. These examples do not arise in connection with lens space surgeries on a knot. Indeed, neither of these knots admits a lens space surgery by Wang and Zhou [29].

Since the trace-field of the knot complements associated to the knots $9_{48}$ and $12 n 642$ is cubic, there are no hidden symmetries (recall Section 2.2), and so the remarks above show that there are finitely many knot complements in this commensurability class (presumably 2).

\subsection{Commensurability classes containing only one knot complement}

A "generic hyperbolic knot" will provide the unique knot complement in its commensurability class. More precisely:

Proposition 5.1 Let $K$ be a hyperbolic knot. If $K$ admits no symmetries, no hidden symmetries, and no lens space surgeries, then $S^{3} \backslash K$ is the only knot complement in its commensurability class. 
Proof Since $K$ admits no symmetries and no hidden symmetries, $S^{3} \backslash K$ is nonarithmetic and will be the minimal element in its commensurability class. Hence any other knot complement commensurable with $S^{3} \backslash K$ covers $S^{3} \backslash K$. This covering is cyclic by [7], and Theorem 3.3 provides a lens space surgery which is a contradiction.

It is conjectured (see Gordon [9]) that if a knot $K$ admits a lens space surgery then $K$ is tunnel number one, so in particular it will admit an order 2 symmetry which is a strong involution. Thus conjecturally any knot without symmetries or hidden symmetries is the only knot complement in its commensurability class.

Example The knot $9_{32}$ provides an example of a hyperbolic knot with no symmetries, no hidden symmetries and no lens space surgeries. Indeed, Riley [23] shows that this knot complement has no symmetries and that its trace field has degree 29. Hence there are no hidden symmetries (see Section 2.2). The computation of the Alexander polynomial shows there are no lens space surgeries by [18].

\subsection{The number of knot complements in a commensurability class}

Based on the above discussion, we have the following conjecture.

Conjecture 5.2 Let $K$ be a hyperbolic knot.

(i) There are at most three knot complements in the commensurability class of $S^{3} \backslash K$.

(ii) If $K$ does not admit symmetries or hidden symmetries then there is only one knot complement in the commensurability class of $S^{3} \backslash K$.

We summarize what is known to us.

Theorem 5.3 Let $K$ be a hyperbolic knot in $S^{3}$. Then Conjecture 5.2 holds for $K$ if one of the following holds:

(i) $K$ is 2-bridge.

(ii) $K$ admits no symmetries, no hidden symmetries and has no lens space surgery.

(iii) $K$ admits a free symmetry but no other symmetries and no hidden symmetries.

(iv) $K$ admits a strong involution but no other symmetries and no hidden symmetries. 
Proof By Theorem 1.1 and Proposition 5.1, it remains to prove (iii) and (iv). For (iii), since the action is free, and there are no hidden symmetries, the minimal orbifold in the commensurability class of $S^{3} \backslash K$ is a manifold, and so this case is handled directly by [20, Theorem 4] and [5].

For case (iv), since $K$ has no hidden symmetries the minimal orbifold $Q_{K}$ (in the previous notation) is $\mathbf{H}^{3} / N(K)$. By assumption, $\left[N(K): \Gamma_{K}\right]=2$. Suppose that $S^{3} \backslash K^{\prime}=\mathbf{H}^{3} / \Gamma_{K^{\prime}}$ is commensurable with $S^{3} \backslash K$, where $\Gamma_{K^{\prime}}<N(K)$. We can assume that $S^{3} \backslash K^{\prime}$ does not cover $S^{3} \backslash K$. For if so, by [28] $S^{3} \backslash K^{\prime}$ cannot cover any other knot complement, and the result follows from [5]. Since the only symmetry of $K$ is a strong involution (which we shall denote by $\tau$ ), $N(K)$ is generated by elements of order 2. Hence the abelianization of $N(K)$ is generated by elements of order 2, and it is easy to see that all elements in the abelianization have order 2. In particular any nontrivial cyclic quotient of $N(K)$ has order 2 .

Since $Q_{K}$ does not have a rigid cusp, Proposition 2.1 shows that $S^{3} \backslash K^{\prime}$ is also a regular cover of $Q_{K}$. Let $G$ be the covering group of $S^{3} \backslash K^{\prime} \rightarrow Q_{K}$. We claim that $G$ is cyclic. Consider the cover $M$ of $S^{3} \backslash K$ and $S^{3} \backslash K^{\prime}$ corresponding to $\Gamma_{K} \cap \Gamma_{K^{\prime}}$. Since $\Gamma_{K^{\prime}}$ is assumed not to be a subgroup of $\Gamma_{K}$, it follows that $M \rightarrow S^{3} \backslash K^{\prime}$ is a 2-fold cover. Hence, $M$ has one cusp. Now $M \rightarrow S^{3} \backslash K$ is also a regular cover, and the covering group is necessarily $G$ (by index). The covering group is abelian since it is determined by the action on the cusp, and hence cyclic since it is the quotient of a knot group. As remarked above, any cyclic quotient of $N(K)$ has order 2 . Since $\mathbf{H}^{3} / N(K)$ has underlying space a ball and ramification locus two arcs labelled 2, there is one 2 -fold cover of $\mathbf{H}^{3} / N(K)$ with a torus cusp (as in the proof of Lemma 2.8 (ii)). Hence $K=K^{\prime}$ in this case.

We have the following corollary of Theorem 5.3(iii).

Corollary 5.4 If $K$ admits no hidden symmetries and has a lens space surgery, then Conjecture 5.2 holds for $K$.

Proof Since $K$ admits a lens space surgery, [29] shows that the only possibility for a nontrivial symmetry of $K$ is a strong involution. Thus either we can apply (iv) of Theorem 5.3, or $S^{3} \backslash K$ is the minimal element in the commensurability class. In which case, any knot complement in the commensurability class covers $S^{3} \backslash K$, and we are done by [7], Theorem 3.3 and [5].

The trace field of the knot complement of the $(-2,3,7)$-pretzel knot has degree 3 . Therefore by Proposition 2.1 it admits no hidden symmetries and there are exactly 3 
knot complements in this commensurability class. It seems likely that there are no examples of knots that have hidden symmetries and admit a lens space surgery.

Since this paper was written, there has been some progress on Conjecture 5.2. In [12], Macasieb and Mattman show that the $(-2,3, n)$ pretzel knots satisfy Conjecture 5.2(i). In [3], Boileau, Boyer and the second author show that Conjecture 5.2(i) is true in the case when $K$ does not admit hidden symmetries.

\section{References}

[1] I R Aitchison, J H Rubinstein, Combinatorial cubings, cusps, and the dodecahedral knots, from: "Topology '90 (Columbus, OH, 1990)", Ohio State Univ. Math. Res. Inst. Publ. 1, de Gruyter, Berlin (1992) 17-26 MR1184399

[2] J Berge, Some knots with surgeries yielding lens spaces, to appear in the proceedings of the Cassonfest

[3] M Boileau, S Boyer, G S Walsh, On commensurability of knot complements, preprint available at http://www.tufts.edu/ gwalsh01/

[4] S Boyer, X Zhang, Finite Dehn surgery on knots, J. Amer. Math. Soc. 9 (1996) 10051050 MR1333293

[5] M Culler, C M Gordon, J Luecke, P B Shalen, Dehn surgery on knots, Ann. of Math. (2) 125 (1987) 237-300 MR881270

[6] W D Dunbar, Hierarchies for 3-orbifolds, Topology Appl. 29 (1988) 267-283 MR953958

[7] F González-Acuña, W C Whitten, Imbeddings of three-manifold groups, Mem. Amer. Math. Soc. 99 (1992) viii+55 MR1117167

[8] O Goodman, D Heard, C Hodgson, Commensurators of cusped hyperbolic manifolds arXiv: 0801.4815

[9] C M Gordon, Dehn filling: a survey, from: "Knot theory (Warsaw, 1995)", Banach Center Publ. 42, Polish Acad. Sci., Warsaw (1998) 129-144 MR1634453

[10] J Hoste, P D Shanahan, Trace fields of twist knots, J. Knot Theory Ramifications 10 (2001) 625-639 MR1831680

[11] H Koch, Number theory, Graduate Studies in Math. 24, Amer. Math. Soc. (2000) MR1760632 Algebraic numbers and functions, Translated from the 1997 German original by $\mathrm{D}$ Kramer

[12] M L Macasieb, T W Mattman, Commensurability classes of $(-2,3, n)$ pretzel knot complements arXiv:0804.0112

[13] C Maclachlan, A W Reid, The arithmetic of hyperbolic 3-manifolds, Graduate Texts in Math. 219, Springer, New York (2003) MR1937957 
[14] G A Margulis, Discrete subgroups and ergodic theory, from: "Number theory, trace formulas and discrete groups (Oslo, 1987)", Academic Press, Boston, MA (1989) 377-398 MR993328

[15] J Milnor, Groups which act on $S^{n}$ without fixed points, Amer. J. Math. 79 (1957) 623-630 MR0090056

[16] W D Neumann, Commensurability and virtual fibration for graph manifolds, Topology 36 (1997) 355-378 MR1415593

[17] W D Neumann, A W Reid, Arithmetic of hyperbolic manifolds, from: "Topology '90 (Columbus, OH, 1990)", Ohio State Univ. Math. Res. Inst. Publ. 1, de Gruyter, Berlin (1992) 273-310 MR1184416

[18] P Ozsváth, Z Szabó, On knot Floer homology and lens space surgeries, Topology 44 (2005) 1281-1300 MR2168576

[19] A W Reid, A note on trace-fields of Kleinian groups, Bull. London Math. Soc. 22 (1990) 349-352 MR1058310

[20] A W Reid, Arithmeticity of knot complements, J. London Math. Soc. (2) 43 (1991) 171-184 MR1099096

[21] R Riley, Parabolic representations of knot groups. I, Proc. London Math. Soc. (3) 24 (1972) 217-242 MR0300267

[22] R Riley, Seven excellent knots, from: "Low-dimensional topology (Bangor, 1979)", London Math. Soc. Lecture Note Ser. 48, Cambridge Univ. Press (1982) 81-151 MR662430

[23] R Riley, Parabolic representations and symmetries of the knot $9_{32}$, from: "Computers in geometry and topology (Chicago, IL, 1986)", Lecture Notes in Pure and Appl. Math. 114, Dekker, New York (1989) 297-313 MR988702

[24] M Sakuma, The geometries of spherical Montesinos links, Kobe J. Math. 7 (1990) 167-190 MR1096689

[25] R E Schwartz, The quasi-isometry classification of rank one lattices, Inst. Hautes Études Sci. Publ. Math. 82 (1995) 133-168 MR1383215

[26] M Suzuki, Group theory. I, Grundlehren series 247, Springer, Berlin (1982) MR648772 Translated from the Japanese by the author

[27] M-o Takahashi, Two-bridge knots have property P, Mem. Amer. Math. Soc. 29 (1981) iii+104 MR597092

[28] S C Wang, Y Q Wu, Any knot complement covers at most one knot complement, Pacific J. Math. 158 (1993) 387-395 MR1206445

[29] S C Wang, Q Zhou, Symmetry of knots and cyclic surgery, Trans. Amer. Math. Soc. 330 (1992) 665-676 MR1031244 
Department of Mathematics, University of Texas Austin, TX 78712, USA

Department of Mathematics, Tufts University

Medford, MA 02155, USA

areid@math.utexas.edu, genevieve.walsh@tufts.edu

http://www.ma.utexas.edu/users/areid/, http://www.tufts.edu/ gwalsh01/

Received: 8 January 2008 Revised: 22 May 2008

Algebraic $\&$ Geometric $\mathcal{T}$ opology, Volume 8 (2008) 